\title{
REDUCING COMPLEXNESS OF CONTROL BY INTELLIGENT MECHANICS IN UNDULANT SWIMMING ROBOTS
}

\author{
M. FREMEREY, L. FISCHHEITER, J. MÄMPEL \& H. WITTE \\ Department of Biomechatronics, Faculty of Mechanical Engineering, \\ Ilmenau University of Technology, Germany.
}

\begin{abstract}
This article introduces a biologically inspired modular swimming robot. Due to defined interfaces in mass, energy, and information flux, the robot's swimming behavior is changeable: an undulant, successive called anguilliform as well as a thunniform swimming mode is adjustable. Unlike the current state of the art, the robot comes with specific designed mechanics for the reduction of the complexity of software-sided control. Thereby, the number of actuators required for propulsion is reduced to the minimum number of one. Currently the prototype robot consists of a basic structure generating amongst others the required torque and several effector modules. The locomotion mode is switchable depending on the number of effector modules. Thereby, the latest anguilliform setup contains three effector modules. The current thunniform configuration features one effector module. The effector modules are mechanically coupled with a manually tunable compliant joint. Optimum values concerning spring stiffness subjected to the location of the joint within the robot are evaluated by simulation. Keywords: aquatic robots, biologically inspired robots, joint with tunable compliance, mechatronic design, modular robot.
\end{abstract}

\section{INTRODUCTION}

For mobile biologically inspired robots, a stable and robust locomotion still poses a challenging aim. Especially while executing difficult tasks in unknown and dangerous environments, the locomotion should be least susceptible to disturbances as possible. To boost reliability of the robot, robustness during the locomotion needs to be increased. This occurred in the past often by the use of a simple mechanical setup (generally a serial connection of motor, gearbox, and shaft) combined with a complex software algorithm. The current article introduces an opposite proceeding: locomotion of the robot is mainly achieved by specific designed mechanics, called 'intelligent mechanics'. Thereby the design of the mechanics may be inspired by motion principles observed from biological species. However, complexity of the control is kept as low as possible.

This approach of reducing control effort by intelligent mechanics is also shown in the researches of Kimura et al [8] and Papadopoulus et al. [13]. In addition, the work of Sproewitz et al. [15] focuses on the energy efficiency of the gait cycles of the light weighted quadruped robot Cheetah. For this purposes, Cheetah features biologically inspired two- and three-segmented legs with a passive, retractable compliance.

The subsequently introduced robot is an aquatic swimming robot. For functional purposes, biological motion principles are transferred. Currently the robot is able to perform two swimming modes of steady swimming fishes: an eel-like anguilliform and a tuna-like thunniform swimming mode. Thereby the generation of propulsion without the commonly used marine propeller enables the exploitation of new application areas in techniques. Ecological sensitive environments become accessible for measurement and task fulfillment. In addition the robot is able to operate in habitats abundantly covered with underwater vegetation like algae. Here the use of a marine propeller would cause failure of the robot: due to the rotational movement, the propeller often gets entangled in the vegetation. In the worst case the motion of the drive shaft is blocked by contamination.

For performing an anguilliform and a thunniform swimming mode, the structure of the robot is a modular one, composing of two different kinds of modules: (i) the basic structure and (ii) the 
effector modules $[1,3,4,6,7,11,12,16]$. The connection among the effector modules is realized by a joint with a manually tunable intrinsic passive compliance. The propulsion of the robot is generated by a single drive. Thereby our approach differs from the current state of the art in modular aquatic robotics, shown in Table 1.

Obviously the design of each joint differs functionally from our approach: ACM-R5, Amphibot 1, and REEL 2 feature joints actuated by a servo-gear combination, the single actuated modular swimming robot has joints with a manually tunable intrinsic stiffness.

On the basis of the results of observations from biology, a modular swimming robot is introduced. For getting important data for the design process, a mathematical model is introduced, which emulates a variant of anguilliform swimming: the slow anguilliform swimming mode. Thereby the model estimates the required driving torque subjected to propulsion. In addition, it enables the investigation of the specific spring stiffness of each joint. On the basis of the results of simulation, the components and modules of the robot are designed. Successive executed experiments show the reliability of the robot.

\section{BIOLOGICAL INSPIRATION}

\subsection{Swimming modes in nature}

In biology, swimming modes of fishes are divisible into two main classes: steady and unsteady swimming fishes (Sfakiotakis et al. [14]). Steady swimming fishes are able to cross long distances with a more or less constant swimming velocity. Their movements are cyclic. The second type contains unsteady swimming fishes. They feature a high maneuverability and are capable as well of quick changes in direction as for rapid starts.

By focusing on steady swimming fishes, four different swimming modes exist (Sfakiotakis et al. [14]): 1. anguilliform, 2. carangiform, 3. thunniform, and 4. ostraciiform. These modes differentiate on the one

Table 1: Comparison of different swimming robots dof(degree of freedom).

\begin{tabular}{|c|c|c|c|c|c|}
\hline Features & & ACM-R5 & Amphi-Bot 1 & REEL 2 & $\begin{array}{c}\text { Modular swimming } \\
\text { robot }\end{array}$ \\
\hline \multirow[t]{2}{*}{ Structure } & setup & modular & modular & $\begin{array}{l}\text { modular with } \\
\text { propulsion fin }\end{array}$ & modular \\
\hline & velocity & $0.4 \mathrm{~m} / \mathrm{s}$ & $0.035 \mathrm{~m} / \mathrm{s}$ & - & $\begin{array}{l}\text { depends on configu- } \\
\text { ration, compare } \\
\text { chapter } 5\end{array}$ \\
\hline \multirow{3}{*}{$\begin{array}{l}\text { Design of } \\
\text { joints }\end{array}$} & actuation & active & active & active & passive \\
\hline & dof & 2 & 1 & 1 & 1 \\
\hline & $\begin{array}{c}\text { kind of } \\
\text { drive }\end{array}$ & $\begin{array}{l}\text { servo for } \\
\text { each dof }\end{array}$ & $\begin{array}{l}\text { servo for } \\
\text { each dof }\end{array}$ & $\begin{array}{l}\text { servo for each } \\
\text { dof }\end{array}$ & $\begin{array}{l}\text { one single servo } \\
\text { actuates the whole } \\
\text { tail structure }\end{array}$ \\
\hline \multirow[t]{2}{*}{ Miscellaneous } & $\begin{array}{l}\text { additional } \\
\text { fins? }\end{array}$ & $\begin{array}{l}\text { for } \\
\text { stabilization }\end{array}$ & not present & $\begin{array}{l}\text { supporting } \\
\text { propulsion }\end{array}$ & $\begin{array}{l}\text { for stabilization and } \\
\text { supporting } \\
\text { propulsion }\end{array}$ \\
\hline & amphibious & yes & yes & no & no \\
\hline
\end{tabular}

ACM-R5 = Hirose et al. [8], Amphibot 1 = Crespi et al. [2], REEL $2=$ Knutson et al. [9] 
hand in the amplitude of the elongated body. It decreases from anguilliform to ostraciiform swimming species. In addition, the animal's relative body length acting against the water is also lowered. On the other hand, flap frequency rises with decreasing amplitude.

\subsection{Assumptions for the design process}

Subsequently a type of the anguilliform swimming mode is investigated: the slow anguilliform swimming mode. Thus the term slow means swimming velocities below one body length per second. The results are carried out from an above-motion tracking of anguilliform swimming species, mainly eels (Laulder et al. [10]). Thereby the swimming animal is reduced to its centerline (aside the spine). The centerline is recorded for the duration of a flap cycle in discrete time lags. Successively, the lines are put on the top of each other. This allows the following conclusions concerning the lateral amplitude of the body subjected to swimming velocity:

While swimming with velocities above one body length per second, eels generate a lateral rhythmic elongation with at least two-thirds of their body. The amplitude of the elongation rises from head to tail; the body is s-shaped. If swimming velocity plunges below one body length per second, the eel only elongates the last third of its body sideways. In addition, the typical s-shape of the body is not formed, the former part of the body hardly moves lateral.

Experiments conducted with fish such as Bass (experimental setup similar to that of eels) with a swimming velocity of 0.7 body lengths per second and 2.4 body lengths per second, have shown that the amplitude of lateral body movement is found to be quite similar in comparison to the slow moving eel (Laulder et al. [10]). In both cases only the last third of the body is laterally elongated. This similarity encompasses the framework for a robot, which is able to perform different swimming modes of steady swimming species. For locomotion purposes the minimum number of a single drive is used.

\subsection{Concept of the modular swimming robot}

The desired investigation of different modes of steady swimming species leads to a modular robot design. It is composed of two types of modules:

1. The basic structure generates the required driving torque. In addition it determines the direction of the robot.

2. The effector modules convert the generated torque into a propulsive force.

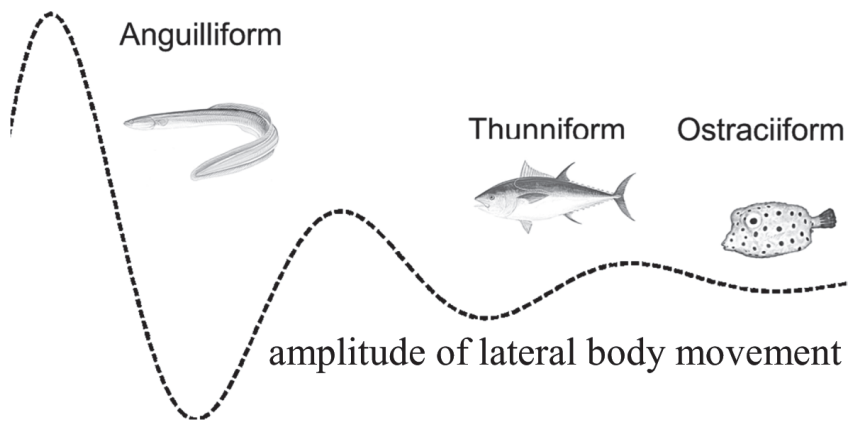

Figure 1: The decline of the amplitude of elongated body from anguilliform to ostraciiform swimming species (Fig. transferred from (Sfakiotakis et al. [14]). 


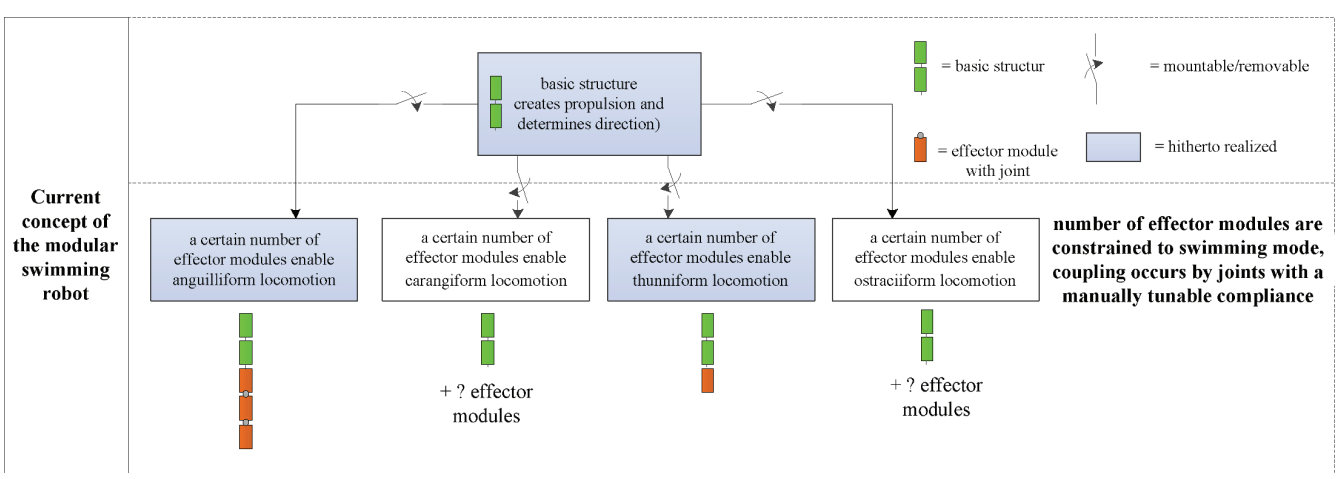

Figure 2: Concept of the modular swimming robot. The four observed swimming modes are realizable by exchanging modules of the robotic platform.

There by the arrangement of the effector modules is a linear one; their number depends on the specific swimming mode. The coupling among them occurs mechanically.

\section{MECHANIC MODEL}

\subsection{Intent of the model}

To obtain data for the constructional process, a mathematical model is introduced. The model centers the gain of propulsion using a slow anguilliform swimming style. The investigation is executed in consideration of two aspects: first aspect is the required driving torque $\mathrm{M}_{\mathrm{a}}$, subject to the prospective propulsive force $\mathrm{F}_{\mathrm{p}}$. The second aspect focuses the influence of the compliance and its adequate description.

\subsection{Model setup}

Keeping the model simple, all unimportant parameters are neglected. Therefore the basic structure is reduced to the driving torque $\mathrm{M}_{\mathrm{a}}$. The effector modules are displayed by several cylindrical segments; these segments compose the tail of the model. Their number and length will be varied during simulation. The continuous compliance of the antetype is transferred to discrete compliant joints. For a further reduction of unknown parameters, the joining among the modules is displayed by a single leg spring.

The model is one side fix-bedded to the ground. A single force considers the flow resistance of the surrounding water. The gravity force is neglected, setting the density of the robot equal to the density of the surrounding fluid, here water. Calculations are executed using the theory of rigid body mechanics: the tail of the robot is modeled as a multi-body system (cf. Fig. 3).

Assuming that the robot is surrounded by static fluid, the force of flow resistance results to

$$
F_{R i}=\frac{1}{2} \cdot \rho \cdot c_{w} \cdot A \cdot v_{i}^{2}
$$




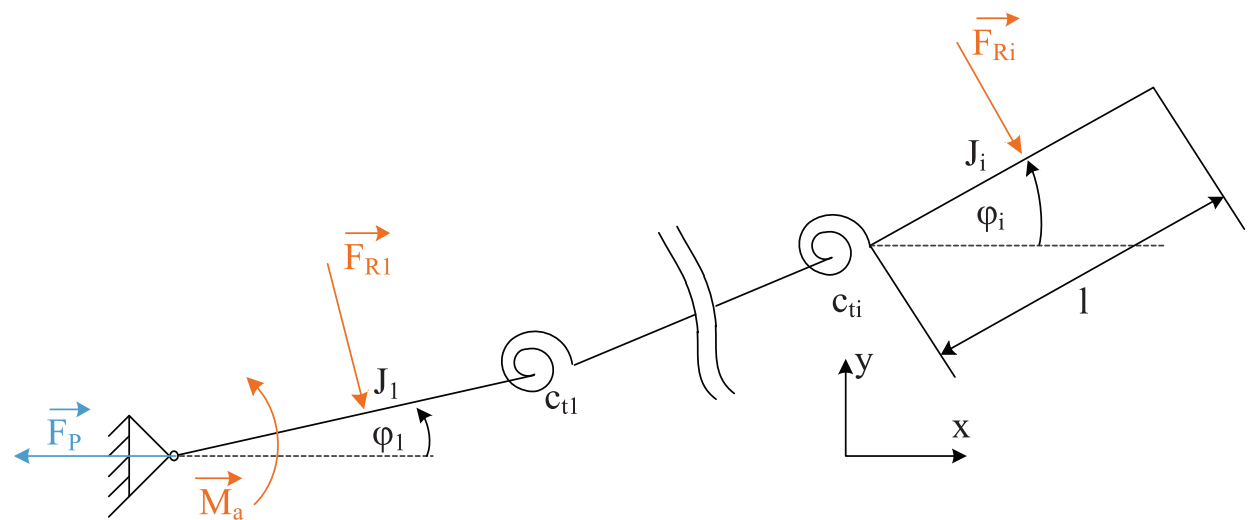

Figure 3: Multi-body model of the tail of the anguilliform swimming robot, $c_{t 1} \ldots c_{t i}=$ spring coefficients, $\phi_{1} \ldots \phi_{\mathrm{i}}=$ elongation of the modules related to a parallel shifted $\mathrm{x}$-axis, $\mathrm{F}_{\mathrm{R} 1} \ldots$ $\mathrm{F}_{\mathrm{Ri}}=$ resistance force of the surrounding fluid, affecting the centre of mass of each effector module, $\mathrm{F}_{\mathrm{p}}$ = gained propulsive force in $\mathrm{x}$-direction, $\mathrm{M}_{\mathrm{a}}=$ applied torque, 1 = length of $\mathrm{a}$ module.

There with the equivalent resistance torque $M_{R i}$ amounts to:

$$
\begin{gathered}
M_{R i}=\frac{l^{3}}{16} \cdot \rho \cdot c_{w} \cdot A \cdot \dot{\varphi}_{i}^{2}=D \cdot \dot{\varphi}_{i}^{2} \\
\text { with } M_{R i}=\frac{l}{2} \cdot F_{R i} \text { and } v_{i}=\frac{1}{2} \cdot \dot{\varphi}_{i}
\end{gathered}
$$

The effective direction of $\mathrm{M}_{\mathrm{Ri}}$ opposite to the direction of the tail movement is considered by the signum function. Due to this consideration, the emulation of damping in the joints becomes negligible. The number of modules is labelled by the subscript i. $\rho$ describes the density of the surrounding fluid (during simulation water). $c_{w}$ is the drag coefficient, A the projected area of the body which resists the water. $\dot{\varphi}$ labels the angle velocity of the elongated body.

$$
M_{R i}=-\operatorname{sign}\left(\dot{\varphi}_{i}\right) \cdot D \cdot \dot{\varphi}_{i}^{2}
$$

The dynamics of a tail with i modules summarizes eqn.(4):

$$
\begin{aligned}
& J \cdot \bar{\varphi}=\sum_{i=1}^{n} m_{i} \\
& J_{1} \cdot \bar{\varphi}_{1}=M_{a}-c_{t_{1}}\left(\varphi_{1}-\varphi_{2}\right)-\operatorname{sign}\left(\dot{\varphi}_{1}\right) \cdot D \cdot \dot{\varphi}_{1}^{2} \\
& J_{2} \cdot \bar{\varphi}_{2}=-c_{t 1}\left(\varphi_{2}-\varphi_{1}\right)-c_{t 2}\left(\varphi_{2}-\varphi_{3}\right)-\operatorname{sign}\left(\dot{\varphi}_{2}\right) \cdot D \cdot \dot{\varphi}_{2}^{2} \\
& J_{i} \cdot \bar{\varphi}_{i}=-c_{t i-1}\left(\varphi_{i}-\varphi_{i-1}\right)-c_{t i}\left(\varphi_{i}-\varphi_{i+1}\right)-\operatorname{sign}\left(\dot{\varphi}_{2}\right) \cdot D \cdot \dot{\varphi}_{2}^{2}
\end{aligned}
$$

Three variants of the mathematical model are investigated. All important data are summarized in Table 2. Varied parameters are number of modules (and therewith the number of joints) and the length of a module. 
Table 2: Features of the simulated models.

\begin{tabular}{lccccc}
\hline Variant no. & No. of modules & $\begin{array}{c}\text { Shape of a } \\
\text { module }\end{array}$ & $\begin{array}{c}\text { Length (mod- } \\
\text { ule) in } \mathrm{mm}\end{array}$ & $\begin{array}{c}\text { Width (module) } \\
\text { in mm }\end{array}$ & No. of joints \\
\hline 1 & 4 & cylinder & 200 & 50 & 3 \\
2 & 3 & cylinder & 200 & 50 & 2 \\
3 & 3 & cylinder & 100 & 50 & 2 \\
\hline
\end{tabular}

\subsection{Transaction of the simulation}

The analysis of the multi-body system uses the software tool ADAMS®. Forcing a motion in the form of a sinusoidal wave to the first effector module enables an oscillation of the tail. Virtual sensors implemented in the model track the required driving torque $\mathrm{M}_{\mathrm{a}}$. Applying the obtained torque $\mathrm{M}_{\mathrm{a}}$ in reverse to the first effector module enables the tracking of the resulting propulsive force $\mathrm{F}_{\mathrm{p}}$.

The suitable spring stiffness of each joint is estimated using a systematic parameter research. The intent of this analysis is the identification of suitable spring stiffness regions by changing the spring stiffness of each joint systematically. By applying a sinusoidal motion to the first effector module, the swinging characteristics of the tail is observed.

\subsection{Results of the systematic research on motion parameters}

The results of each variant are illustrated in Fig. 4. The sinusoidal function for stimulating the model illustrates eqn. (5). $t$ labels the time, $\mathrm{w}$ a weighting factor.

$$
f=\sin \left(\frac{t}{4}\right) \cdot w
$$

The diagrams in Fig. 4 reveal three states concerning the swinging behavior of the tail.

State 1): The value of the spring stiffness is located in the range of possible spring stiffness (dashed line, dashed-dotted line). Within this range, the tail oscillates with a smooth swinging characteristic close to the biological antetype. Thereby each joint has a theoretical optimum of spring stiffness.

State 2): Adjusting the spring stiffness below the range of possible spring stiffness: due to the tiny spring stiffness, the tail oscillates with raising amplitude. At a certain point, the hypercritical gain causes a flipover of individual modules. This could cause damage or in the worst case a destruction of the whole tail structure.

State 3): Adjusting the spring stiffness above the range of possible spring stiffness: the segmented tail acts like a single rigid body. In this state no elongation of single modules is observable; the spring stiffness is chosen to be too stiff.

During simulation, variant 2 achieves best results concerning a smooth swinging behavior. The optimum values for spring stiffness amount $1 \cdot 10^{-3} \mathrm{Nm} \cdot \mathrm{deg}^{-1}$ (joint one) and $1 \cdot 10^{-4} \mathrm{Nm} \cdot \mathrm{deg}^{-1}$ (joint two). This configuration requires a driving torque of $0.02 \mathrm{Nm}$; therewith a propulsive force of about $0.02 \mathrm{~N}$ is theoretically achievable. As a result of the positive sign of $\mathrm{F}_{\mathrm{p}}$, a forward motion is gained. 

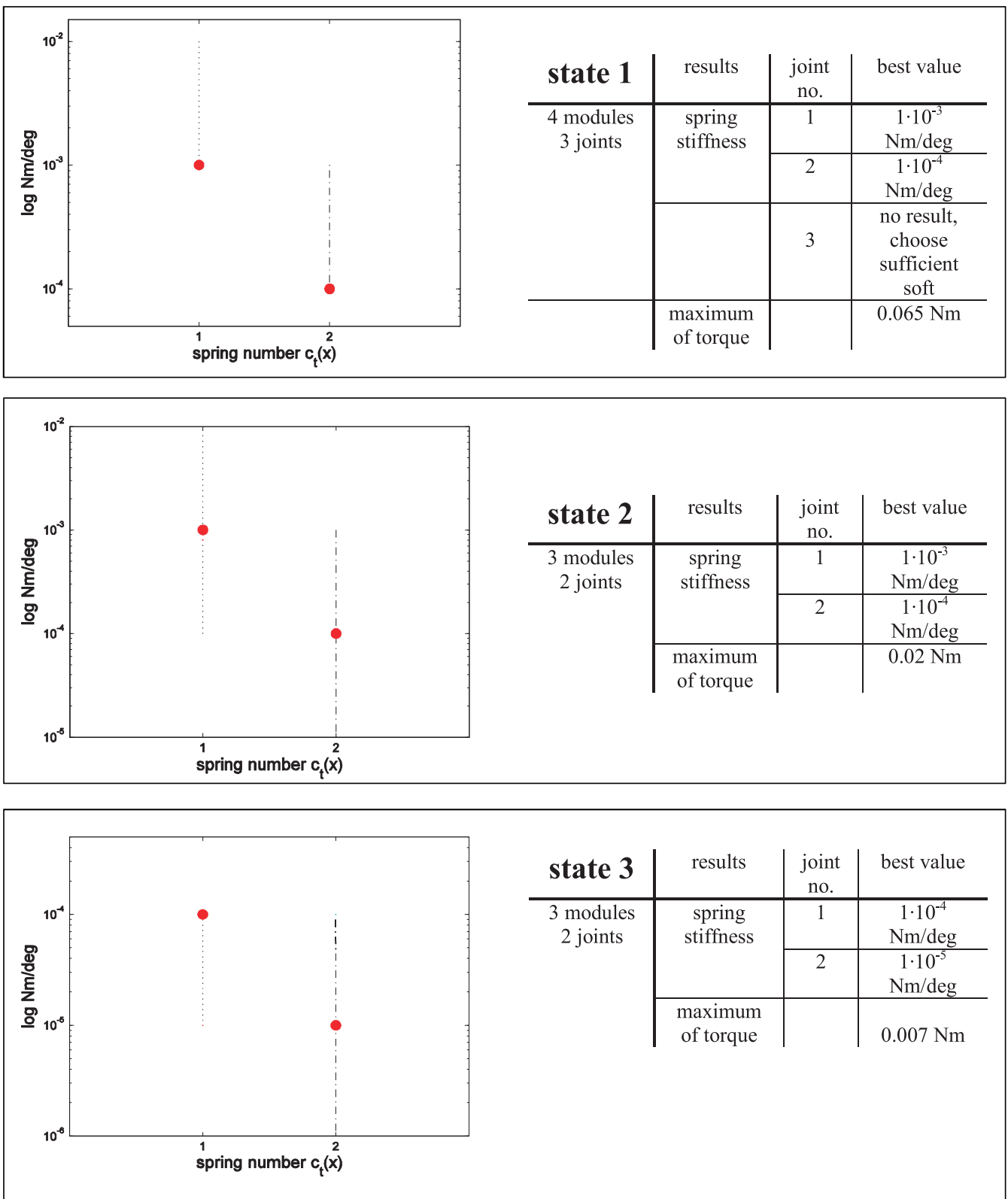

Figure 4: Simulation results of the systematic parameter research. For each variant the range of possible spring stiffness is plotted (dashed line, dashed-dotted line). Red dots represent a theoretical optimum of spring stiffness, the variant shows best swinging performance. Beyond this range, single effector modules either flip over (spring stiffness too soft) or have no compliant swinging characteristic (spring stiffness too tough). The tables aside the plots summarize most important data gained by the simulation concerning each variant. 
4 DESIGN OF THE MODULAR SWIMMING ROBOT

Each module of the robot consists of a cylindrical tube with a length of $200 \mathrm{~mm}$ and an inner diameter of about $50 \mathrm{~mm}$. The applied material is acrylic glass due to its resistance against salt water and to aesthetic reasons. Each tube is sealed by two acrylic glass caps. A greased gasket along with a bolted connection prevents water leakage. The required technical elements for locomotion and task fulfillment are placed on insertion plates within the modules.

\subsection{Design of the modules of the basic structure}

The basic structure implements two main tasks: (i) creating the required torque for locomotion purposes and (ii) determining the swimming direction of the robot.

An actuator-gear combination provides the required torque. The actuator is a modified common servo drive (HSR-5980SG HiTEC enterprise, Germany). It has a maximum of torque of about $3 \mathrm{Nm}$. The gear is a self-made cam mechanism. It translates the continuous rotational movement of the servo drive into an alternating one. The amplitude of this oscillation is about \pm 15 degree, derived from the results of the simulation. Thereby the gear also reduces the complexity of the control structure: the translation from continuous to alternating rotation is only done by mechanics.

A change in the swimming direction of the robot is done by a thin metallic sheet mounted to a rotatable lever. The helm is elongated by another servo drive (BMS 303, Bluebird enterprise). Thereby the rotatable lever is embedded into a stern tube construction for avoiding water penetration. Aside the motor control and the energy supply are fixed inside the basic structure, as well as the receiver for the wireless transmitted control signals.

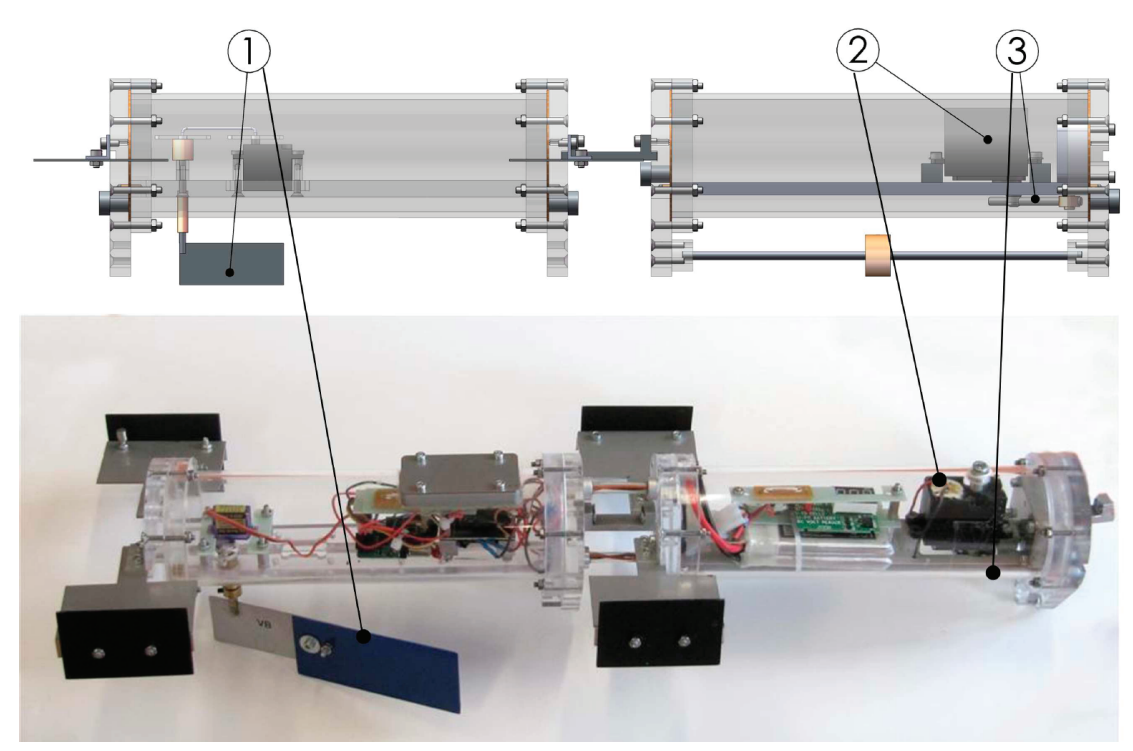

Figure 5: CAD drawing and design of the basic structure, $1=$ helm, $2=$ servo drive, $3=$ self-made cam mechanism. The basic structure also contains the energy supply, the cruise control, and the receiver required for the remote control. 
4.2 Design of the effector modules and the joints with a manually tunable passive compliance

The effector modules transform the generated torque to a propulsive force. Their number depends on the desired swimming mode. Between the effector modules, the joint with the manually tunable passive compliance enables the desired oscillating swinging characteristic. This joint consists of a revolute joint which ensures a rigid connection of the modules among each other. The intrinsic compliance of the joint is realized by linear spring elements that replace the rotational springs used during simulation. Therewith an easy technical implementation is facilitated. The algorithm of this conversion is described in Fremerey et al. [5] in detail.

To counter changing conditions of aquatic environment, the so-called extrinsic compliance, linear spring elements feature a manual calibration: sliding the bedding of the elements to the left or to the right changes the lever ratio: the spring stiffness is changed.

Currently two configurations consisting of different effector modules are investigated. The first configuration is based on the results of the executed simulation. Here the slow anguilliform swimming mode is transferred to techniques by the use of three effector modules. Due to the results of simulation, the stiffness is reduced from head to tail by a factor of 10 concerning each joint. Conveying the rotational stiffness into a linear one (Fremerey et al. [5]), the stiffness amounts $77.5 \mathrm{~N} \cdot \mathrm{m}^{-1}$ for joint one (starting head side) and $7.75 \mathrm{~N} \cdot \mathrm{m}^{-1}$ for joint two.

The second configuration transfers the principles of thunniform locomotion: a single effector module is used here.

\subsection{Electronics and control}

The current version of the robot is remotely controlled by the user. Swimming only at the water surface, a standard wireless communication operating at $40 \mathrm{MHz}$ is adequate. A cruise control

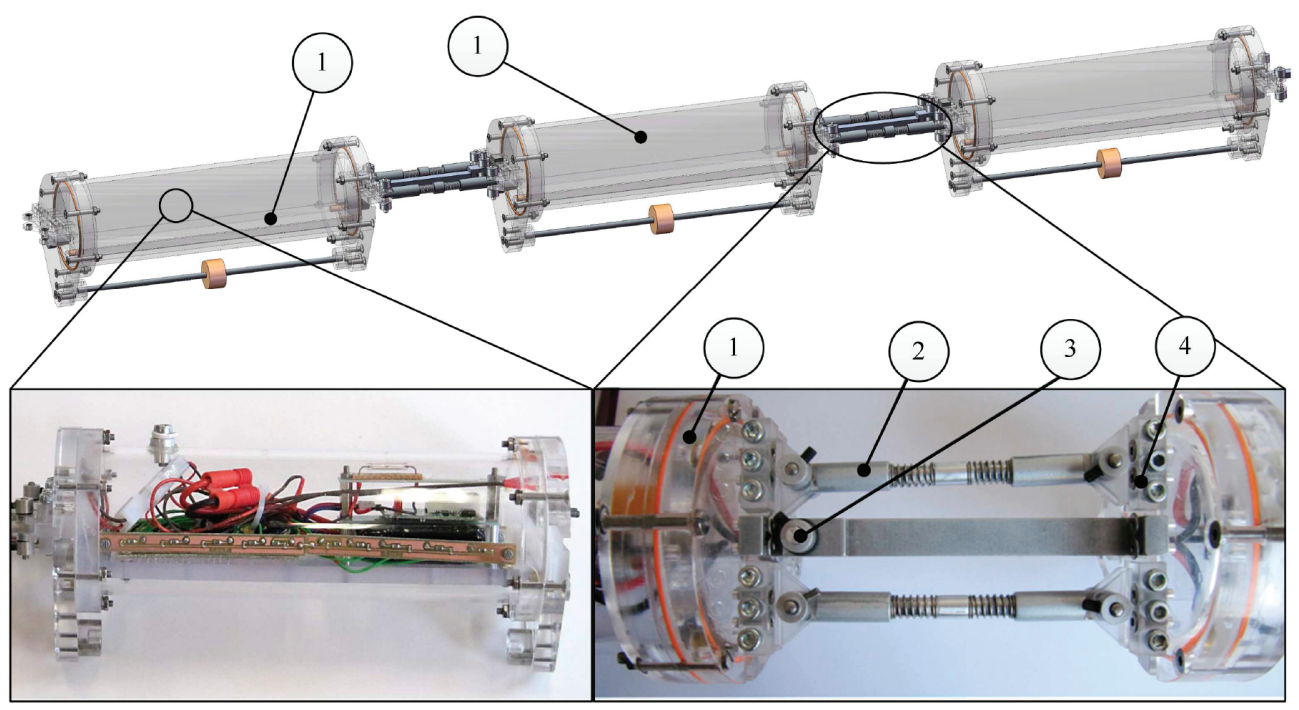

Figure 6: CAD drawing of effector modules and designed joint with manually tunable passive compliance;, 1 = effector module, 2 = linear spring element, 3 = rigid revolute joint, $4=$ slidable bedding of the spring elements. 
alienates the signals from the receiver to the drive. Due to a hitherto not implemented sensory system, voltage of the drive and the position of the helm are controlled in an open loop. The energy is provided by onboard Lithium-Polymer accumulators due to their high energy density.

\section{EXPERIMENTS}

Subsequently both possible configurations are assembled to the basic structure; the swimming velocity is estimated. The configurations are tested without and with a fin connected to the last effector module. Due to the length of the robot, experiments are executed in a real environment, here an artificial lake at TU Ilmenau. During the test, wind velocity was negligible. Figure 7 illustrated the results of the swimming test for the anguilliform configuration (configuration A).

Configuration A features three mechanically coupled effector modules. During experiments swimming velocity ranges from $0.01 \mathrm{~m} \cdot \mathrm{s}^{-1}$ up to $0.02 \mathrm{~m} \cdot \mathrm{s}^{-1}$. Although adding a flexible fin (material: FR4, length: $190 \mathrm{~mm}$, width: $45 \mathrm{~mm}$, thickness: $0.5 \mathrm{~mm}$ ) to the last effector module, swimming velocity remains in the range of $0.01 \mathrm{~m} \cdot \mathrm{s}^{-1}$ to $0.02 \mathrm{~m} \cdot \mathrm{s}^{-1}$. However the anguilliform configuration delivers a forward motion. Comparable to the results of propulsion gained during simulation, the velocity is quite slow. The reasons for the uniformity of the velocity have to be investigated in future: executing a systematic parameter research on range of adjustable spring stiffness may reveal more beneficial values than the current ones.

Configuration B contains the thunniform setup. The experiments are also executed in the artificial lake at TU Ilmenau. Having only a single effector module, the swimming velocity amounts $0.02 \mathrm{~m} \cdot \mathrm{s}^{-1}$ up to $0.03 \mathrm{~m} \cdot \mathrm{s}^{-1}$. Adding a fin having the same dimensions used during the experiments of the anguilliform configuration raises the velocity to $0.08 \mathrm{~m} \cdot \mathrm{s}^{-1}-0.1 \mathrm{~m} \cdot \mathrm{s}^{-1}$. The thunniform configuration also achieves a forward motion of the robot.

In addition, the thunniform configuration shows a quite good maneuverability. The implementation of the helm to the basic structure enables a change in direction of the robot to the left and the right. The current radius of a curved trajectory amounts about $1.5 \mathrm{~m}$ to $2 \mathrm{~m}$. However, adjusting the anguilliform configuration, a change in direction of the robot is not possible any more: the robot just moves forward. This constraint is going to be one of the future tasks.

\section{FUTURE TASKS}

By executing some first swimming experiments, additional experimental setups for further evaluation and knowledge gain are planned, e.g. the systematic evaluation of suitable spring stiffness for each joint within the real robot. Another possible setup consists of a motion analysis of the whole robot using a tracking software tool, e.g. 'MaxTRAQ 2d'. Those experiments will deliver information

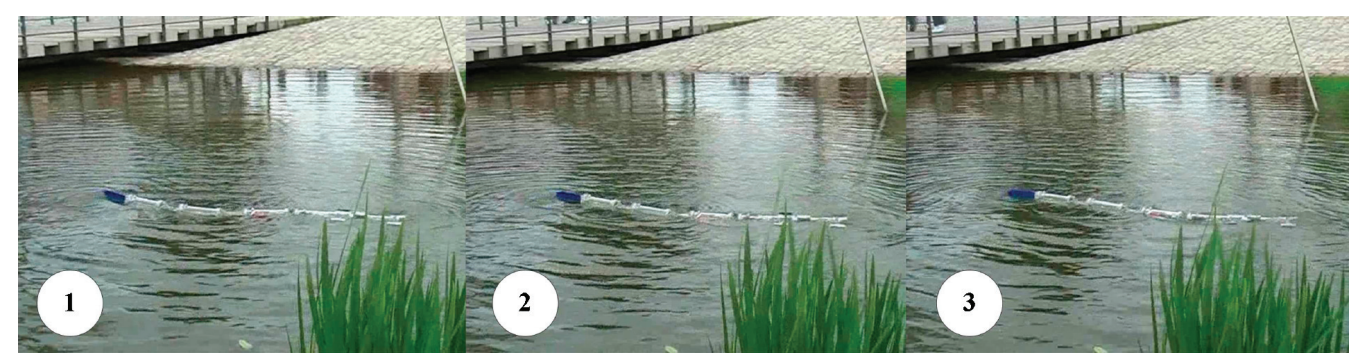

Figure 7: Executed swimming test, configuration A (anguilliform). Frames are taken every two seconds. 
about the motion of the effector modules via several swimming cycles. The results of both experiments are also usable for improving the quality of the introduced mathematical model. Another possible task is the tracking of the power consumption of the swimming robot.

To improve the design of the robot, the joint with tunable passive compliance is going to be further developed. Being now only manually tunable, the compliance will be set in future by the usage of adjusting drives. They will support the single drive for locomotion as well in guiding and adapting the robot to different environments. In addition, a shift between different swimming modes is achievable in real-time, e.g. from anguilliform to thunniform swimming.

Thereby, the range of possible applications will be further enlarged. One possible example is the investigation of oil films after a shipwreck: due to the use of the real-time adjustable compliance in each joint, the robot changes its swimming mode and pattern subject to the circumstances of environment. Task fulfillment in liquids with different viscosity, e.g. water and oil becomes feasible.

\section{CONCLUSIONS}

On the basis of the results of the investigation of the swimming modes of steady swimming fishes, a modular swimming robot is introduced. In contrast to the current state of the art, the robot features only a single drive for locomotion purposes. The propulsion is generated by a tail that consists of a variable number of effector modules. Due to the modular setup the robot is able to perform an anguilliform and a thunniform swimming mode.

For the anguilliform swimming mode, a mathematical model is introduced. It evaluates necessary data for construction, like generated propulsion subject to the required driving torque. During

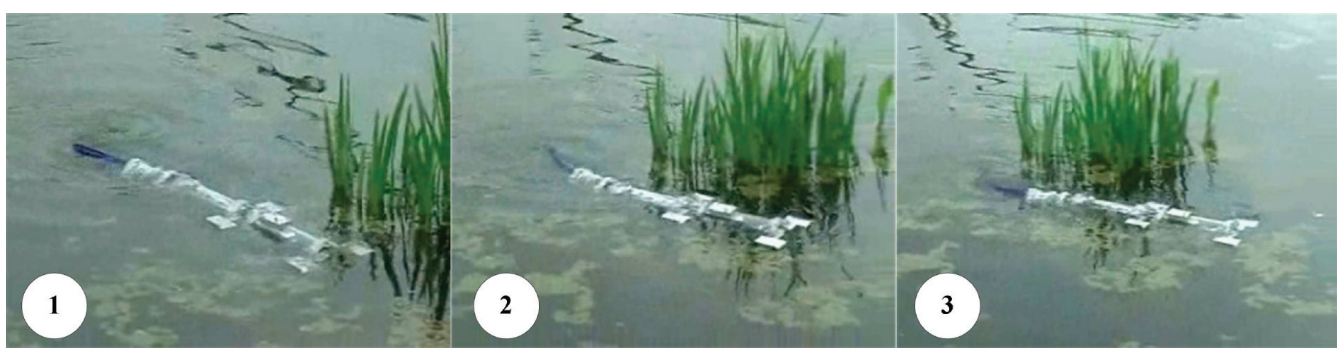

Figure 8: Executed swimming test, configuration B (thunniform). Frames are taken every two seconds.

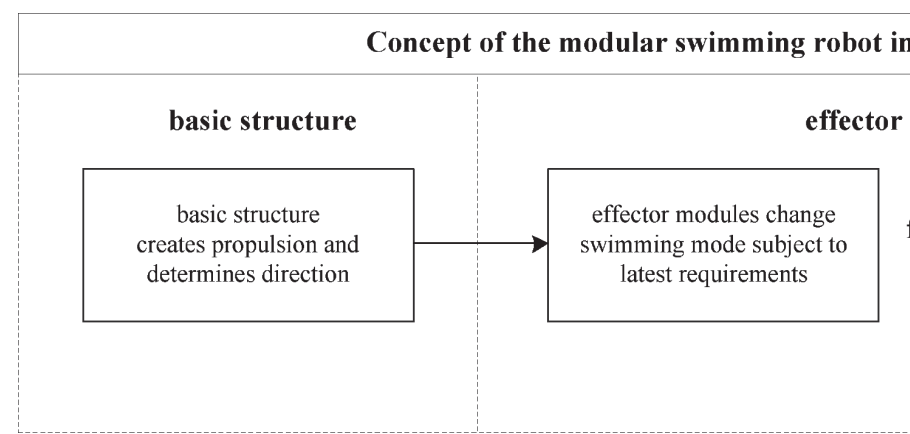

Figure 9: Concept of the modular swimming robot in the future. 
simulation a tail consisting of three effector modules showed best swinging performance. The coupling between the modules occurs by using rotational springs, a systematic parameter research ascertains the optimum values for the stiffness. Due to an easier technical implementation, the rotational springs were converted to linear compression springs during the constructional process.

Currently the robot swims at a velocity of $0.01 \mathrm{~m} \cdot \mathrm{s}^{-1}$ to $0.02 \mathrm{~m} \cdot \mathrm{s}^{-1}$ (with and without a fin) while featuring the anguilliform configuration. The thunniform configuration enables a swimming velocity of $0.02 \mathrm{~m} \cdot \mathrm{s}^{-1}$ to $0.03 \mathrm{~m} \cdot \mathrm{s}^{-1}$ without and $0.08 \mathrm{~m} \cdot \mathrm{s}^{-1}$ to $0.1 \mathrm{~m} \cdot \mathrm{s}^{-1}$ with a fin. In both cases a stable forward motion is achieved by the use of intelligent mechanics. The control is reduced to an open loop wireless, one controlling the voltage of the drive and the position of the helm-steering servo.

\section{ACKNOWLEDGMENT}

We thank Danja Voges for her support in literature research in the biological fields and photography. A special thank goes to Eberhard Hamatschek and Michael Bastick for their support in the workshop during this project.

\section{REFERENCES}

[1] Brüggen, N., Technik der U-Boot Modelle. Verlag für Technik und Handwerk: Baden-Baden, 1992.

[2] Crespi, A., Badertscher, A., Guinard, A. \& Ijspeert, A., AmphiBot 1: an undulant snake-like robot. Robotics and Autonomous Systems 50, pp. 163-175, 2005. doi: http://dx.doi.org/10.1016/j. robot.2004.09.015

[3] Fremerey, M., Fischheiter, L., Mämpel, J. \& Witte, H. Design of a single actuated, undulant swimming robot. The 3rd International Symposium on Mobiligence, Awaji, Japan, pp. 174-178, 2009.

[4] Fremerey, M., Fischheiter, L., Mämpel, J. \& Witte, H.. Locomotion study of a single actuated, modular swimming robot. 5th Confernce on Design and Nature, Pisa, Italy, pp. 138-150, 2010.

[5] Fremerey, M., Fischheiter, L., Mämpel, J. \& Witte, H., Locomotion study of a single actuated, undulant swimming robot. Proceedings for the joint conference of ISR 2010 (4 ${ }^{\text {st }}$ International Symposium on Robotics) and ROBOTIK 2010 (6th German Conference on Robotics), Munich, Germany, CD-ROM, 2010.

[6] Graham, J.B. \& Lowell, W.R., Surface and subsurface swimming of the sea snake pelamis platurus. Physiological Research Laboratory and Marine Biology Research Division: Scripps Institution of Oceanography, University of California, San Diego, USA, 1986.

[7] Hirose, S. \& Yamada, H., Snake-like robots - machine design of biologically inspired robots. IEEE Robotics \& Automation Magazine, pp. 88-98, 2009. doi: http://dx.doi.org/10.1109/ MRA.2009.932130

[8] Kimura, H., Fukuoka, Y. \& Cohen, A., Biologically inspired adaptive walking of a quadruped robot. Phiosophical Transactions of the Royal Society, 365(1850), pp. 153-170, 2007.

[9] Knutsen, T., Ostrowski, J. \& McIsaac, K., .Designing an Underwater eel-like Robot and Developing Anguilliform Locomotion Control. NSF Summer Undergraduate Fellowship in Sensor Technologies: Tamara Harvard University, pp. 119-142.

[10] Laulder, G.V. \& Tytell, E.D., Hydrodynamics of undulatory propulsion. Fish Biomechanics, Vol. 23, 2005.

[11] Mämpel, J., Eisold, R., Kempf, W., Schilling, C. \& Witte, H., A modular concept for a biologically inspired robot. Robot Motion and Control, pp. 391- 400, 2009. 
[12] Mämpel, J., Koch, T., Koehring, S., Obermaier, A. \& Witte, H., Concept of a modular climbing robot. IEEE Symposium on Industrial Electronics and Applications (ISIEA), Kuala Lumpur, Malaysia, 2009. doi: http://dx.doi.org/10.1109/ISIEA.2009.5356362

[13] Papadopoulos, D. \& Buehler, M. Stable running in a quadruped robot with compliant legs. IEEE International Conference on Robotics and Automation, (ICRA), Vol. 1, pp. 444-449, 2000.

[14] Sfakiotakis, M., Lane, D.M. \& Davies, J.B., Review of fish swimming modes for aquatic locomotion. IEEE Journal of Oceanic Engineering, 24, pp. 237-252, 1999. doi: http://dx.doi. org/10.1109/48.757275

[15] Sproewitz, A., Fremerey, M., Karakasiliotis, K., Rutishauser, S., Righetti, L. \& Ijspeert, A., Compliant leg design for a quadruped robot. Abstracts of Dynamic Walking 2009. Vancouver, Canada, 2009.

[16] Zhang, H.X., Gonzalez-Gomez, J., Chen, S.Y., Wang, W., Liu, R., Li, D. et al., A Novel Modular Climbing Catapillar Using Low-frequency Vibrating Passive Suckers, Proceeding of AIM, 2007. 